\title{
AppEAR: Una aplicación móvil de ciencia ciudadana para mapear la calidad de los hábitats acuáticos continentales
}

\author{
JoAquín Cochero \\ Instituto de Limnología "Dr. Raúl A. Ringuelet" - Facultad de Ciencias Naturales y Museo, Universidad Nacional de La Plata- \\ CONICET
}

\begin{abstract}
Resumen. La ciencia ciudadana, o ciencia participativa, es el proceso a través del cual el público en general contribuye activamente a proyectos científicos. En las últimas décadas, los proyectos de ciencia ciudadana proliferaron gracias a Internet y están demostrando ser muy útiles para complementar los monitoreos ambientales "tradicionales". AppEAR ("app" para la Evaluación de Ambientes Ribereños) tiene como objetivo contribuir al mapeo del estado de los ambientes acuáticos continentales, con énfasis en el hábitat de sus riberas y costas, dentro de un marco de ciencia ciudadana colaborativa. Consiste en una aplicación móvil gratuita que permite a los usuarios evaluar el hábitat de ríos, arroyos, lagos y estuarios desde dispositivos móviles, a través de cuestionarios y fotografías. La aplicación también incluye material educativo y lúdico para diferentes edades, relacionado con las características y el cuidado de los ecosistemas acuáticos de aguas continentales. En su primer año, el proyecto AppEAR incorporó $\sim 460$ usuarios que aportaron información sobre 131 sitios muestreados en todo el país. A través de sus contribuciones, los ciudadanos científicos reconocieron las problemáticas ambientales más comunes, que incluyen el uso del suelo que rodea al sitio, la calidad de la vegetación de ribera y la presencia de basura. Aunque es importante conocer y reconocer las limitaciones del uso de herramientas digitales masivas para recolectar datos con fines científicos, se las debe entender como un complemento de las técnicas tradicionales de monitoreo, que pueden enriquecer las distintas etapas de los estudios de investigación.
\end{abstract}

[Palabras clave: Android, app, ciudadano científico, educación ambiental]

\begin{abstract}
AppEAR: A citizen science mobile app to map the habitat quality of continental waterbodies. Citizen science, or participatory science, is the process by which the general public actively contributes with scientific projects. Citizen science projects have proliferated in the few last decades thanks to the Internet and are proving to be a great complement to the more "traditional" environmental monitoring efforts. AppEAR (an app to evaluate the aquatic habitats) states as its main objective to map the status of the habitat of continental waterbodies, with a primary focus on its shores and coasts, within a citizen science framework. It consists of a free mobile app that allows users to evaluate the habitat quality of rivers, streams, lakes and estuaries from their mobile devices, by means of questionnaires and photographs. The app also includes educational material and games, related to the characteristics and caring of the continental waterbodies. In its first year, the AppEAR project incorporated about 460 users who sent information of nearly 131 sampling sites throughout the country. Through their efforts, citizen scientists reported as their main environmental concerns the degradation of the land use around the sites, the quality of the riparian vegetation and the presence of debris or garbage. Even though it is important to recognize the limitation of these digital tools for massive data recollection for scientific purposes, they should be considered as a complement for the more traditional monitoring efforts, that can enrich the different stages in research projects.
\end{abstract}

[Keywords: Android, app, citizen science, environmental education]

\section{INTRODUCCIÓN}

La ciencia ciudadana, o ciencia participativa, es el proceso a través del cual el público en general contribuye de forma activa con proyectos científicos, sin necesariamente ser científicos profesionales (Haklay 2015). Esta metodología está demostrando ser muy útil para complementar las posibles limitaciones de los monitoreos ambientales "tradicionales", es decir, aquellos que científicos y agencias gubernamentales suelen llevar a cabo coordinadamente con herramientas estandarizadas (Kruger and Shannon 2000; Conrad and Hilchey 2011; Klemann-Junior et al. 2017). Según las características del proyecto, estas limitaciones pueden incluir complicaciones en la recolección de grandes volúmenes de datos, en escalas espaciales pequeñas $y$, a veces, con grandes costos económicos (Cohn 2008; Dickinson et al. 2010; Tulloch et al. 2013). 
A pesar de que las observaciones de los naturalistas amateurs fueron importantes por muchos siglos, en las últimas décadas proliferaron los proyectos de ciencia ciudadana proliferaron, con la habilidad de seguir los cambios en impactos sociales y ecológicos a grandes escalas espaciales y temporales a través de Internet (Lepczyk et al. 2009). Aplicaciones sofisticadas en línea emplean de manera efectiva el concepto de colaboración abierta distribuida ("crowdsourcing") para recolectar datos a través de grandes regiones geográficas y ofrecen a los participantes la oportunidad de proveer, acceder e interpretar los datos de manera colectiva (Dickinson et al. 2010). Tecnologías asociadas a los dispositivos móviles a través de aplicaciones (del inglés, "apps"), redes de sensores inalámbricos y computación y juegos en línea se muestran como una gran promesa para avanzar en lo que sedenomina "ciencia ciudadana digital" (Burke et al. 2006). La incorporación del concepto de "gamificación" (del inglés, "gamification", que es el uso de las mecánicas del juego, su estética y el pensamiento para involucrar a la gente, motivar la acción, promover el aprendizaje y resolver problemas [Kapp 2012]) a los proyectos de ciencia ciudadana digital puede motivar a los participantes y atraer a nuevos usuarios (Bowser et al. 2014).

Los datos producidos por "ciudadanos científicos" (también llamados científicos amateurs o voluntarios) no sólo pueden contribuir mucho a los programas de monitoreo ecológico, sino también integrar a los usuarios en el proceso de generación de conocimiento científico (Irwin 2001; Cooper et al. 2007). De esta manera, los participantes acceden a materiales y protocolos de enseñanza, colectan datos, los ingresan a bases de datos centralizadas e, incluso, contribuyen a validarlos y analizarlos. A su vez, de manera abierta pueden ver los resultados de los demás participantes a través de gráficos y mapas interactivos. La ciencia ciudadana participa, además, en la educación de nuevos actores, en la concienciación social sobre los componentes ambientales, en el empoderamiento de las acciones individuales ante el fenómeno y en la demanda del accionar político-económico para incluir conceptos ambientales en agendas gubernamentales (Conrad and Hilchey 2011).

Los retos asociados con la ciencia ciudadana se pueden resumir en tres categorías: de organización, de recolección de datos y de uso de datos (Conrad and Hilchey 2011). Entre las problemáticas de organización se mencionan el financiamiento (Whitelaw et al. 2003), el reclutamiento de ciudadanos científicos (Conrad and Daoust 2008), el acceso a la información (Milne et al. 2006) y la generación de redes de trabajo (Milne et al. 2006). En el caso de la recolección de datos, los problemas más comunes incluyen la fragmentación de datos, su falta de precisión y la falta de objetividad de los participantes (Whitelaw et al. 2003), principales consecuencias de la variabilidad en la habilidad, experiencia y entrenamiento de los participantes (Fitzpatrick et al. 2009; Dickinson et al. 2010). Por ello, la validación tanto de los datos individuales como del conjunto de datos debe incluir en alguna etapa a personal entrenado o profesional. Por último, los datos obtenidos en proyectos de ciencia ciudadana no siempre se alcanzan a usar en instancias de toma de decisiones o en artículos científicos (Milne et al. 2006; Conrad and Daoust 2008), y es frecuente que los investigadores no realicen intentos de corregir los errores de muestreo (Lavado Contador 2005; Conrad and Hilchey 2011).

Cabe también considerar que los proyectos digitales requieren que la usabilidad y el diseño sean efectivos y que la cantidad de información que se le pide al usuario sea limitada, por lo general basada en íconos y gráficos más que en la entrada de texto. Esto puede limitar su uso, pero obliga también a los investigadores a pensar críticamente sobre qué información es esencial y cuál es suplementaria $\mathrm{u}$ opcional. Los objetivos del proyecto deben ser concretos y bien definidos.

\section{El PROYECTO ApPEAR}

El proyecto AppEAR ("app" para la Evaluación de Ambientes Ribereños) tiene como objetivo principal contribuir al mapeo del estado de los ambientes acuáticos continentales, con énfasis en el hábitat de sus riberas y costas, dentro de un marco de ciencia ciudadana. Para alcanzarlo se propuso desarrollar una aplicación móvil gratuita que permita a sus usuarios evaluar el hábitat de dichos ecosistemas (ríos, arroyos, lagos, estuarios) a través de un cuestionario simple y de la observación del ambiente que los rodea, sin necesidad de dispositivos de muestreo u observación sofisticados. La aplicación también incluye material educativo y lúdico para diversas edades, relacionado con las características y el cuidado de los ecosistemas acuáticos de aguas continentales. 
Como resultados esperados del proyecto se buscará recaudar información sobre cuatro categorías principales: 1) usos del suelo de la zona circundante al cuerpo de agua, 2) características de la vegetación arbórea y acuática, 3) características del agua (color, olor, transparencia, velocidad) y 4) características físicas de las márgenes (pendiente, residuos, canalizaciones, etc.). Esta información permitirá ir generando, de manera colectiva y colaborativa, una línea de base del estado de los ecosistemas acuáticos continentales.

La información recolectada a través de la plataforma es dispuesta en formato abierto y de libre acceso en el sitio web del proyecto. Estos datos se podrán usar sin restricciones por otros científicos, organismos de gestión y educativos para diversos propósitos, como para la generación de índices ecológicos o estudios de líneas de base de ecosistemas acuáticos.

\section{LA APLICACIÓN MÓVIL ApPEAR}

Consiste de dos secciones básicas: un cuestionario y la toma de fotografías. Los datos recolectados por medio de un dispositivo móvil son enviados a un servidor vía Internet, que los muestra en un mapa virtual de libre acceso. Además de poseer una finalidad científica, la aplicación tiene un componente educativo (con textos, figuras, infografías sobre los ecosistemas acuáticos y juegos) y un componente lúdico, a través de un sistema de puntos y recompensas virtuales.

\section{Construcción de la aplicación}

La aplicación fue generada para dispositivos con sistema operativo Android ${ }^{\circledR}$ por medio del software Anywhere Software B4A Enterprise Edition (Licencia B4X170716-9984-49115). Este soft permite la codificación en lenguaje Basic (Visual Basic .NET) y exporta la compilación en

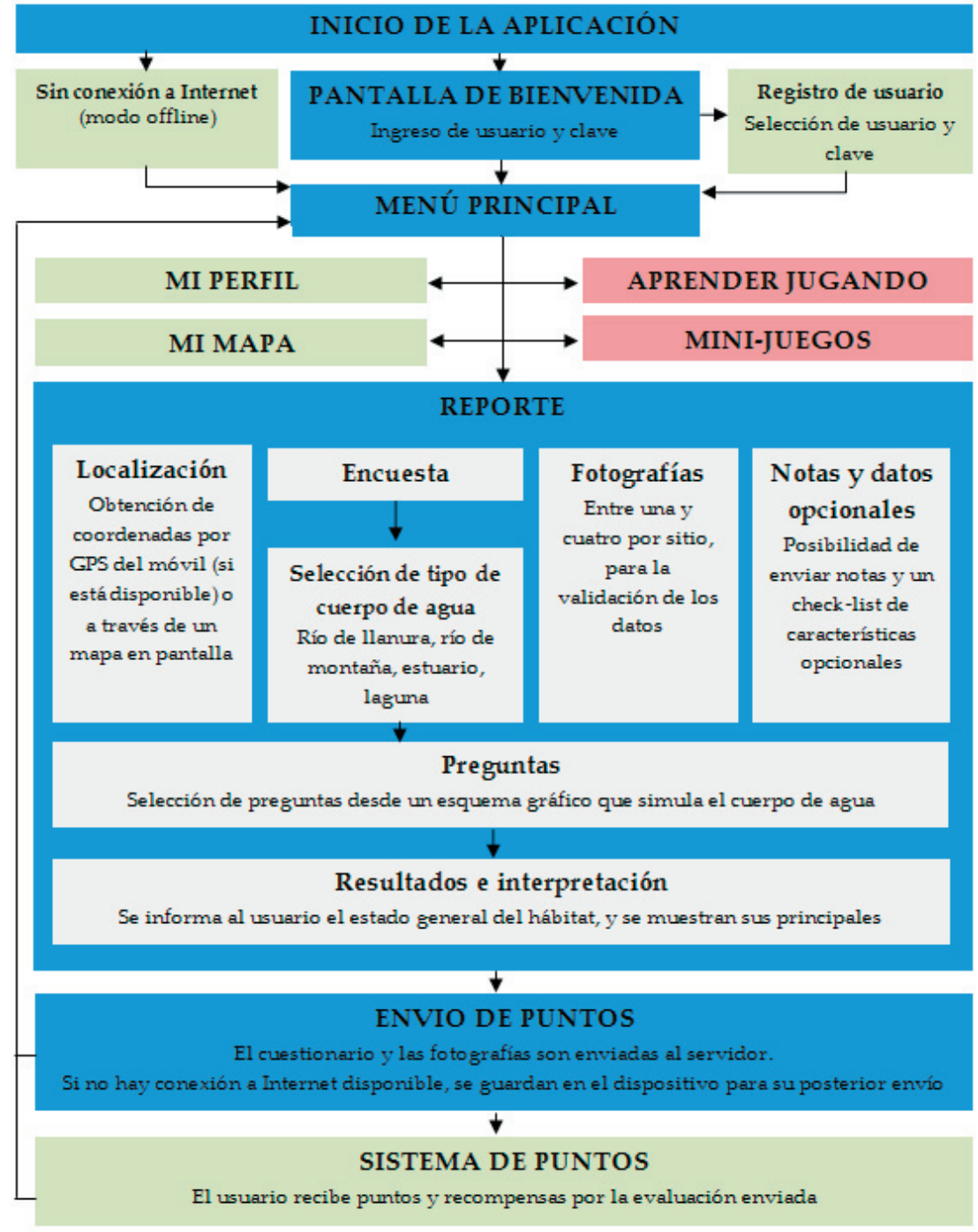

Figura 1. Diagrama de bloques del funcionamiento de la aplicación móvil AppEAR. Los bloques en azul indican la trayectoria desde el inicio de la aplicación hasta el envío de un reporte completo. Los bloques de color rojo demarcan las características educativas de la aplicación, mientras que los de color verde indican las funciones accesorias al funcionamiento.

Figure 1. Functioning diagram of the app. Blue blocks indicate the trajectory from the startup of the app until the data is sent. Red blocks indicate the educational features while green blocks indicate accessory functionalities. 
lenguaje Java para ser utilizado en dispositivos con sistema operativo Android $\AA$.

El flujo de la aplicación (Figura 1) comienza con la solicitud al usuario de sus credenciales (nombre de usuario y clave). Si el usuario no posee dichas credenciales, puede registrarse desde la pantalla inicial completando un formulario, siempre y cuando el dispositivo posea de conexión a Internet. También dispone de la opción de seleccionar entre idioma español e inglés, lo cual permite captar un público objetivo más amplio al subir la aplicación a diversos catálogos de proyectos de ciencia ciudadana online (e.g., scistarter.com).

\section{Menú principal}

Desdeel menú principal, el usuario tiene cinco opciones: 1) evaluar un sitio (el comienzo de la evaluación de un sitio), 2) mi mapa (el usuario puede ver los sitios que envió anteriormente en un mapa), 3) mi perfil (el usuario puede acceder y modificar su información, así como enviar al servidor las encuestas que no envió anteriormente), 4) aprender jugando (contiene textos informativos y juegos educativos de dificultad intermedia, referidos a la contaminación, el ciclo hidrológico, las redes tróficas y las comunidades acuáticas) y 5) mini-juegos (este ítem comprende juegos sencillos para niños [juego de memoria, juego de palabras]).

\section{La evaluación de un sitio}

La evaluación de un sitio requiere de manera obligatoria tres aspectos: a) establecer la localización geográfica del sitio a evaluar, b) tomar al menos una fotografía del sitio y c) completar una encuesta. Además, se permite la adición de información opcional en forma de notas y comentarios. Para establecer la localización geográfica del sitio a evaluar se muestra un mapa virtual en la pantalla; allí, el usuario puede seleccionar manualmente su posición, o de manera automática por medio del GPS incorporado en el dispositivo.

Las fotografías se pueden tomar con la cámara del dispositivo o se pueden adjuntar las almacenadas. Por cada sitio evaluado, la aplicación permite el envío de hasta cinco fotografías. Para cada foto, un texto en la aplicación orienta al usuario en cómo tomar la fotografía para que la revisión del dato sea más sencilla (tomar dos fotos del sitio en general, desde distintos ángulos, y las restantes tres con características distintivas como la cantidad de residuos o la vegetación).

La encuesta varía según el tipo de ambiente a evaluar en cuatro categorías: ríos de montaña, ríos de llanura, lagunas y estuarios, aunque posee la capacidad de incorporar otros ambientes en actualizaciones posteriores. Para cada ambiente se utilizó un cuestionario que incluye unas preguntas generales y preguntas particulares para cada ambiente. Las preguntas generales fueron redactadas sobre la base de los formularios propuestos en el manual de monitoreo rápido de cursos de agua (US EPA 1997), de los cuales se seleccionaron aquellas de sencilla evaluación visual que no requiriesen de una capacitación adicional de los ciudadanos científicos. Para determinar cuáles preguntas resultaban más fáciles para los usuarios, en una primera instancia se realizaron talleres entre profesionales, y en una segunda etapa se utilizaron los grupos de prueba descriptos más adelante. El usuario tiene, además, la opción de agregar comentarios y notas adicionales, así como también puede completar una "check-list" para indicar la presencia de los organismos más frecuentes en los ambientes acuáticos (e.g., aves, peces, anfibios, etc.).

\section{Las encuestas}

Las preguntas de la encuesta se disponen de manera secuencial mediante una interfaz gráfica que simula el ambiente acuático elegido y que se modifica según las respuestas del usuario. Las preguntas se clasifican en cuatro categorías: usos del suelo, vegetación, características del agua y modificaciones de la morfología del tramo (ver lista completa de preguntas por tipo de ambiente en el Anexo I). Para cada ambiente se ideó un sitio de buena calidad del hábitat y se consideró que las desviaciones de esta situación ideal disminuirían el valor de calidad del hábitat en mayor o menor medida

Con respecto a los usos del suelo, se consideraron como de mejor calidad aquellas zonas de reserva o zonas naturales poco alteradas, con ausencia de corrales ganaderos de engorde ("feedlots") o con poco ganado disperso, ya que su presencia puede favorecer la eutrofización (Carpenter et al. 1998). Se consideraron también como elementos que disminuyen la calidad del hábitat la presencia de basura (e.g., los plásticos actúan como contaminantes [Wagner et al. 2014; Dris et al. 2015]) y de escombros en las riberas (e.g., 
González and Tánago 2011; Belmar et al. 2013). Asimismo, se consideraron como perjudiciales para la calidad del hábitat la presencia de murallas bordeando los cuerpos de agua, ya que reducen la continuidad natural entre estos ambientes y promueven cambios en el desarrollo de comunidades acuáticas (Francis and Hoggart 2009). La presencia de muelles y edificaciones en la ribera también reducen el valor de calidad, ya que los motores de botes y automóviles generan contaminantes que pueden infiltran hacia los cuerpos de agua (e.g., Liddle and Scorgie 1980).

En cuanto a la vegetación de ribera se consideraron como indicadores de mejor calidad la presencia de vegetación de algún tipo (lo cual favorece el "efecto filtro" [Daniels and Gilliam 1996; Tabacchi et al. 2000b; Anbumozhi et al. 2005]); se otorga un mayor valor de calidad si la vegetación presente es nativa. Asimismo, la cobertura parcial por sombreado de la vegetación ribereña genera parches (luzsombra) que aumentan la heterogeneidad del hábitat acuático; sombreados intermedios demostraron aumentar la diversidad del cauce (Dawson and Kern-Hansen 1979; Tabacchi et al. 2000a). También se considera en la encuesta la presencia de plantas acuáticas, juncos o pajonales, ya que, además de proveer una mayor diversidad de hábitats para los organismos acuáticos, pueden actuar absorbiendo nutrientes y metales pesados ( Miretzky et al. 2004; Mishra and Tripathi 2008).

Con respecto a las preguntas relacionadas a las características del agua se incluyeron aquellas vinculadas al olor, a la transparencia y al color. Aguas transparentes son normales en ambientes de montaña o lagunas, mientras que aguas amarronadas por sedimentos en suspensión son comunes en ríos de llanura. El agua con colores oscuros pueden implicar alto contenido de materia orgánica y sustancias húmicas (Steelink 1977; Nürnberg 1996; Steinberg 2013).

Entre las modificaciones de la morfología natural del tramo, el usuario puede indicar si el sitio se encuentra desbordado o en su cauce natural, si se encuentra canalizado o entubado, si sus márgenes son muy inclinadas y estimar la heterogeneidad del fondo. El desborde de los cursos de agua que naturalmente no incorporan esa dinámica suele ser un indicador de impedimentos hidráulicos que entorpecen el flujo normal, y su canalización o entubamiento reduce tanto la diversidad de hábitats acuáticos como la conexión del curso con el ambiente hiporréico (Lake et al. 2000; Malmqvist and Rundle 2002; Langhans et al. 2008). En particular en arroyos de llanura, las márgenes muy empinadas pueden ser producto de su dragado (Hupp 1992), que es una práctica de manejo de gran impacto sobre la biodiversidad acuática (Strayer 2006). La cantidad de sustratos diferentes en el fondo de los cuerpos de agua también es considerada como una medida de heterogeneidad de ambientes (Cardinale et al. 2010). El usuario puede indicar si ve arcillas, arenas, piedras o gravas y guijarros, o si es un fondo artificial (e.g., cemento).

\section{Calidad del hábitat y envío de información}

Una vez finalizada la encuesta se expone un mensaje informativo del estado general del hábitat del sitio (muy bueno, bueno, moderado, malo, muy malo), así como una lista con las posibles variables que resultan en la degradación de ese ambiente acuático. El valor de calidad del hábitat se calcula como el promedio de los valores asociados a cada pregunta para cada tipo de cuerpo de agua (Anexo; Tabla 1). Si el dispositivo móvil tiene conexión a Internet, el usuario puede enviar los resultados al servidor. Si no se puede conectar, la evaluación queda guardada en el dispositivo para su posterior envío.

\section{Calidad de los datos colectados}

Los datos enviados por los ciudadanos científicos son inicialmente considerados como "no validados", es decir, son aquellos datos crudos que no pasaron por un proceso de revisión por personal entrenado o científico. La revisión de calidad se realiza desde una interfaz dorsal (o "back-end") desde un sitio web, sólo accesible por los revisores. Desde la interfaz dorsal, los revisores pueden ver las respuestas a los cuestionarios junto con las fotografías enviadas y revisar cada dato individual. Si la fotografía coincide con lo expresado en el cuestionario en todas sus variables, el revisor cambia de estado a "validado".

\section{Validación de los datos}

Se realizaron dos validaciones de los datos enviados por los usuarios. La primera comprendió una validación entre lo que enviado por ciudadanos científicos y lo enviado por profesionales entrenados en el estudio de los ecosistemas acuáticos, ambos 
utilizando la aplicación. El objetivo de esta validación fue comprobar la claridad en la utilización del aplicativo y de las encuestas; de esta manera se evalúan las posibles diferencias entre un usuario con entrenamiento científico formal y el público en general. Para ello se seleccionaron 30 sitios (Anexo 2) que fueron evaluados tanto por profesionales científicos como por usuarios amateurs, y con los valores obtenidos de calidad del hábitat para cada sitio se realizó una prueba de t de a pares.

La segunda etapa de validación tuvo como objetivo evaluar si los valores de calidad de hábitat obtenidos a través de las encuestas en la aplicación correlacionaban significativamente con índices ecológicos del hábitat ya establecidos. Para ello se seleccionaron 18 datos de arroyos de la provincia de Buenos Aires y 18 datos del estuario del Río de la Plata (Anexo 2) y se los comparó con los valores obtenidos para esos mismos sitios a través de dos índices ecológicos del hábitat (índice USHI [Cochero et al. 2016] e índice IHRPlata [Gómez and Cochero 2013]). Estos índices usan parámetros similares a los medidos en la aplicación para calcular un valor único de calidad del hábitat de ribera en arroyos urbanos y periurbanos (USHI) y del hábitat de la costa del estuario del Río de la Plata (IHRPlata), y fueron desarrollados como herramientas sencillas para evaluar el hábitat de ambientes ribereños y estuariales, ya que correlacionan significativamente con diversos parámetros químicos y biológicos de la calidad del agua (i.e., concentraciones de nutrientes, de oxígeno disuelto, turbidez, conductividad). Para establecer las relaciones entre los valores de calidad del hábitat obtenidos por los índices bióticos y los obtenidos en la aplicación se realizaron correlaciones de Pearson. La validación de los datos obtenidos en ambientes de montaña y lagunares, etapa en proceso actualmente, aún no se ha realizado por faltante de datos para contrastar aquellos obtenidos por los usuarios desde la aplicación.

\section{Disposición y visualización de la información}

El proyecto dispone de la información de manera libre y abierta. Para ello se generó un sitio web (www.app-ear.com.ar) que contiene todos los datos enviados por los ciudadanos científicos, visibles en un mapa que se actualiza en tiempo real. También permite filtrar aquellos reportes validados de los no validados por profesionales científicos, y descargar las bases de datos de los datos del proyecto. En el sitio web también se puede ver un listado de los ciudadanos científicos que más contribuyeron con el proyecto.

\section{Gamificación}

La aplicación móvil aquí presentada incluye una mecánica de puntos y recompensas. Al finalizar el envío de cada evaluación, los usuarios ganan puntos y medallas virtuales que pueden ser compartidas en las redes sociales. La acumulación de puntos por los ciudadanos científicos puede ser visualizada desde el sitio web del proyecto, y podrá ser utilizada en eventos específicos, tales como competencias o eventos educativos masivos.

\section{Privacidad de los usuarios}

Durante el registro de usuarios se le solicita el ingreso obligatorio de un nombre de usuario y una clave, junto con sus nombres, lugar de origen, fecha de nacimiento y el tipo de perfil de usuario (profesional científico, amateur, docente, alumno de escuela). Toda la información es asegurada en una base de datos MySQL con clave de doce dígitos, y las claves de los usuarios son encriptadas por el sistema MD5 para que no sean visibles por los administradores del sitio web. La única información expuesta en el sitio web de manera pública es el nombre de usuario.

\section{Grupos de prueba y lanzamiento}

Previo a su lanzamiento masivo se conformaron grupos de prueba de la aplicación para revisar que su funcionamiento fuera adecuado y que las preguntas de los cuestionarios fueran comprensibles y no requirieran de una mayor capacitación de los usuarios. Un grupo de prueba fue conformado por profesionales científicos del Instituto de Limnología "Dr. Raúl A. Ringuelet"; otro, por niños entre cinco y quince años de edad relacionados con el Club "El Retiro" (partido de La Plata). La aplicación fue lanzada a través del sistema Google Play en junio de 2016.

\section{Resultados}

\section{Los usuarios de AppEAR}

En su primer año, la aplicación AppEAR ha incorporado 460 usuarios, que han enviado información sobre 131 sitios muestreados. De los usuarios registrados, el 50.7\% lo hicieron indicando un perfil de "profesional científico"; un $31.8 \%$ indicó un perfil relacionado a la 
educación (docentes, alumnos) y el restante $17.5 \%$ indicó un perfil "amateur", no vinculado a las otras dos categorías.

De los 131 datos enviados hasta la fecha, el $41.2 \%$ correspondieron a datos de arroyos de llanura, $23.6 \%$ a arroyos de montaña, el $16.0 \%$ a datos de estuarios, y el restante $19 \%$ a lagunas.

\section{Mapa del hábitat}

En el mapa virtual disponible en el sitio web del proyecto se muestran todos los puntos enviados, con la posibilidad de filtrar aquellos datos validados (Figura 2). En el mapa se indica el estado general del hábitat en los distintos colores de los marcadores (azul=muy bueno; verde=bueno; amarillo=regular; rojo=malo). $\mathrm{Si}$ se selecciona un punto, se muestran los detalles sobre el reporte y el nombre del usuario que contribuyó con el envío. Dentro de Argentina, la mayor cantidad de datos pertenecieron a sitios dentro de la provincia de Buenos Aires (69.2\%), seguido por Río Negro (7.1\%) y Tierra del Fuego (5.8\%). Las provincias de Córdoba, Neuquén, Santa Cruz, Corrientes, Jujuy y Salta contribuyeron con porcentajes menores a $2 \%$ de los datos. Los registros de otros países alcanzaron $7.7 \%$ de los datos.

\section{Validación de los datos}

Los resultados de la prueba comparativa entre los datos obtenidos por ciudadanos científicos y profesionales para los mismos sitios de muestreo no mostraron diferencias significativas entre los valores de calidad del hábitat obtenidos por ambos grupos (prueba de $\mathrm{t}$ a pares, $\mathrm{t}=0.634 ; P=0.52 ; \mathrm{n}=30$ ). En los arroyos de llanura periurbanos se encontró una correlación significativa entre los valores de calidad del hábitat obtenidos por ciudadanos científicos desde la aplicación y los calculados a través del índice de calidad del hábitat (USHI, Pearson $\mathrm{R}=0.668, P<0.01$, $\mathrm{n}=18)$. En el caso de los datos de calidad del hábitat obtenidos en ambientes de estuarios se encontró una correlación significativa entre los medidos a través de la aplicación y los calculados con el índice de calidad del hábitat (IHRPlata, Pearson $\mathrm{R}=0.603, P<0.01$, $\mathrm{n}=18)$.

\section{Problemáticas ambientales más comunes}

Con los resultados de las encuestas de las preguntas generales a los cuatro ambientes se pudo reconocer las problemáticas del hábitat más comunes percibidas por los ciudadanos científicos. Entre los descriptores del hábitat

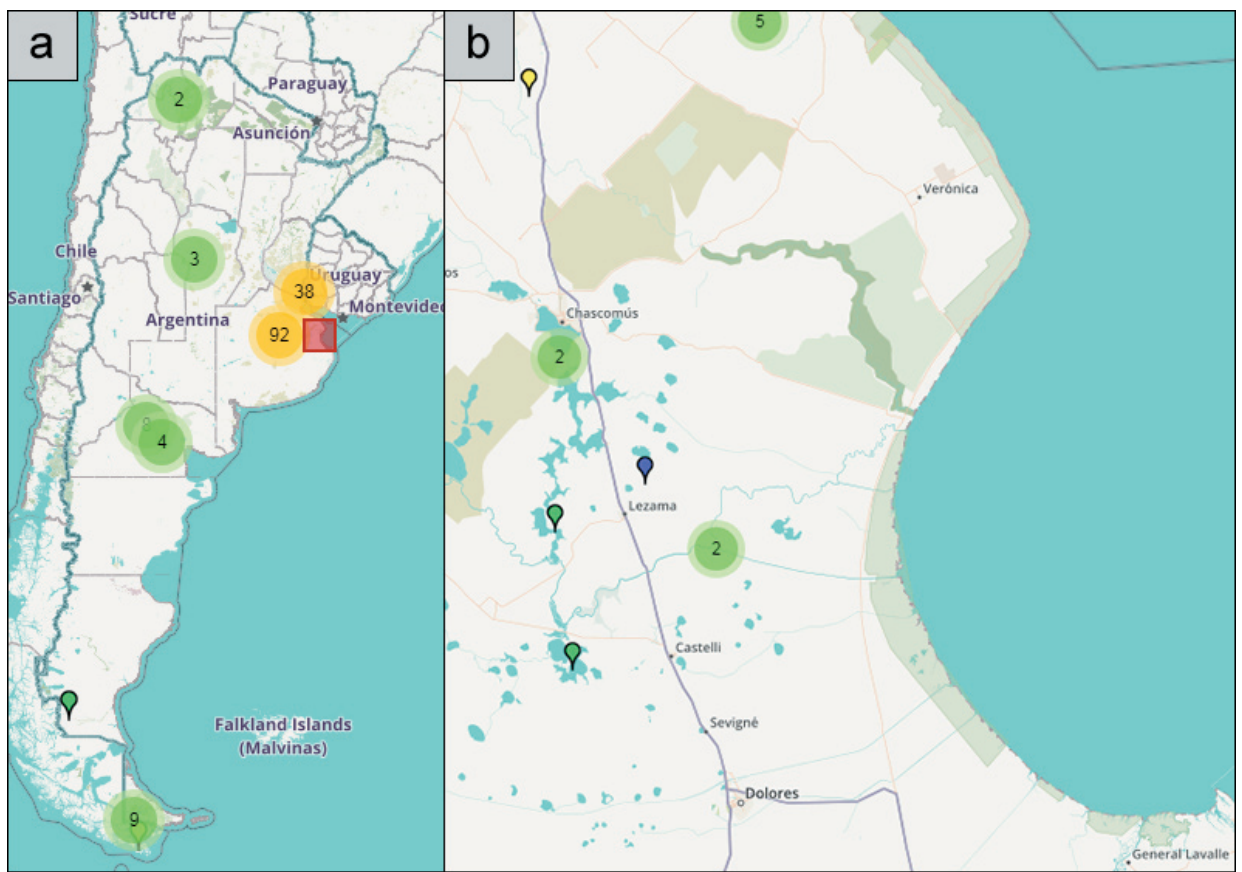

Figura 2. a) Mapa de la Argentina con los reportes enviados por los ciudadanos científicos en el primer año de proyecto. b) Ejemplo de detalle de la zona cercana a la Bahía de Samborombón (provincia de Buenos Aires). Los colores de los marcadores indican la calidad general del hábitat del punto evaluado (azul=muy bueno; verde=bueno; amarillo=regular; rojo=malo).

Figure 2. a) Map of Argentina marking the reports sent by citizen scientist throughout the first year of the project. b) Detailed area near the Samborombón Bay (province of Buenos Aires) as an example. The color of the markers indicates the overall quality of the habitat for each site (blue=very good; green=good; yellow=regular; red=bad). 
Evaluación general de todos los sitios $(n=131)$

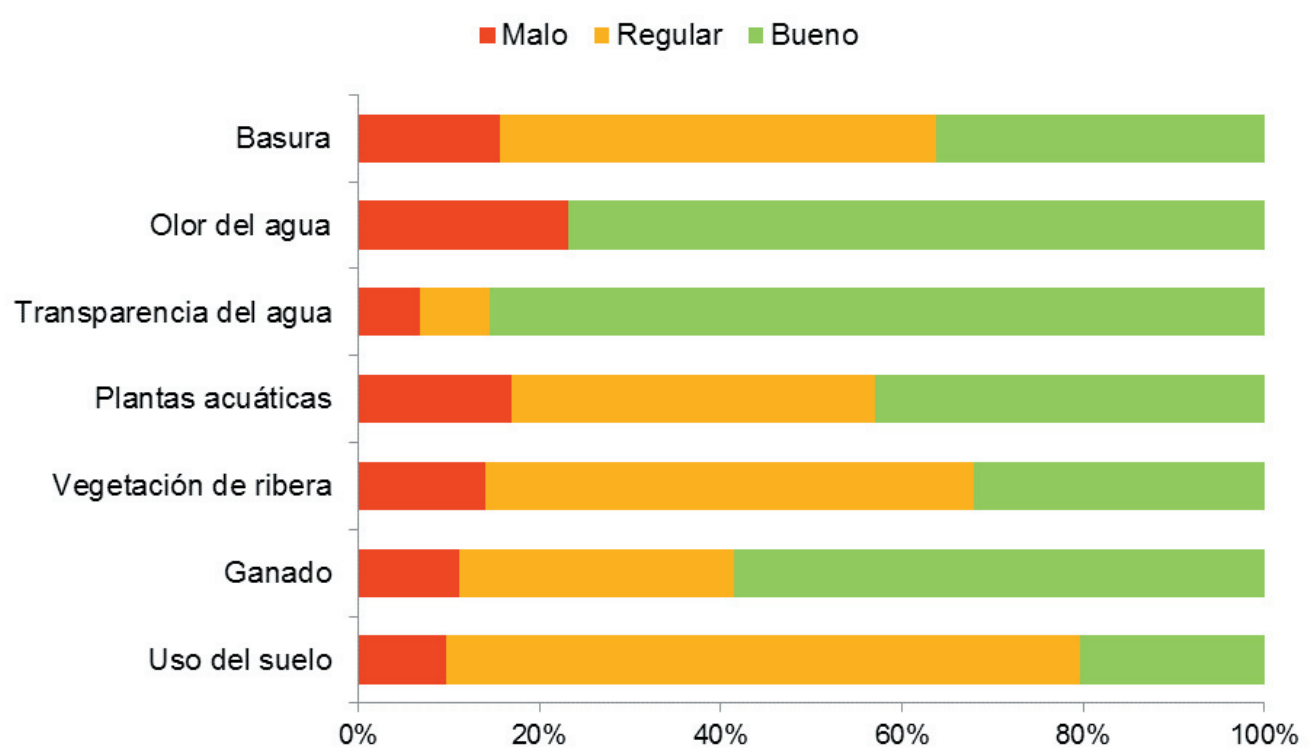

Figura 3. Variables comunes a todos los ambientes evaluados por los ciudadanos científicos, y su porcentaje de reportes malos, regulares y buenos.

Figure 3. Common variables to all the environments sampled by citizen scientists and their percentages of bad, regular and good reports.

con mayor cantidad de casos malos y regulares se destacan el uso del suelo que rodea al sitio, la calidad de la vegetación de ribera y la presencia de basura (Figura 3). Entre los arroyos de montaña y llanura, los resultados evidencian una baja heterogeneidad del fondo y una alta degradación de los márgenes en aquellos sitios de llanuras (Figuras 4). En los estuarios, las variables con más casos con estado malo incluyen la presencia de escombros, de muelles y murallas, de degradación de los pajonales costeros y de basura (Figura 4). En las lagunas, las variables más afectadas implican la presencia de muelles y murallas.

\section{DisCUSIÓN}

Los monitoreos realizados por ciudadanos científicos contribuyen a generar y reconocer patrones a gran escala espacial. Sus resultados son útiles para influenciar políticas a niveles nacionales e internacionales. A escalas de manejo locales, el monitoreo realizado por organismos científicos suele ser incorporado más lentamente en las políticas de manejo que aquellas llevadas a cabo por líderes comunitarios en conjunto con la comunidad local. A estas escalas puede ser muy beneficioso el desarrollo de programas de monitoreo participativo, ya que las comunidades se reconocen inmersas en la problemática ambiental (Danielsen et al. 2010).

El desarrollo y la difusión de AppEAR permitió explorar su potencial para obtener gran cantidad de datos a grandes escalas geográficas y en relativamente poco tiempo. Durante el primer año de su funcionamiento se sumaron 131 reportes del estado del hábitat de diversos ambientes de todo el país, tarea poco factible de ser realizada en ese tiempo con muestreos tradicionales. Un tercio de los usuarios que descargó la aplicación se adjudicó un rol en la educación (docentes y alumnos). Esto introduce temáticas tales como las características y el cuidado del hábitat acuático en el ambiente educativo en diversas etapas, aunque el posible impacto del proyecto en dicho ambiente no puede ser cuantificado de una forma tan directa como el aspecto científico.

Ciertamente, los desafíos de la ciencia ciudadana son varios, no sólo su etapa de organización, sino también de calidad de los datos y de utilización de los mismos (Conrad and Hilchey 2011). En las pruebas comparativas realizadas, los datos que obtuvieron los ciudadanos científicos y los científicos profesionales no difirieron 
AppEAR, CIENCIA CIUDADANA EN ECOSISTEMAS ACUÁTICOS

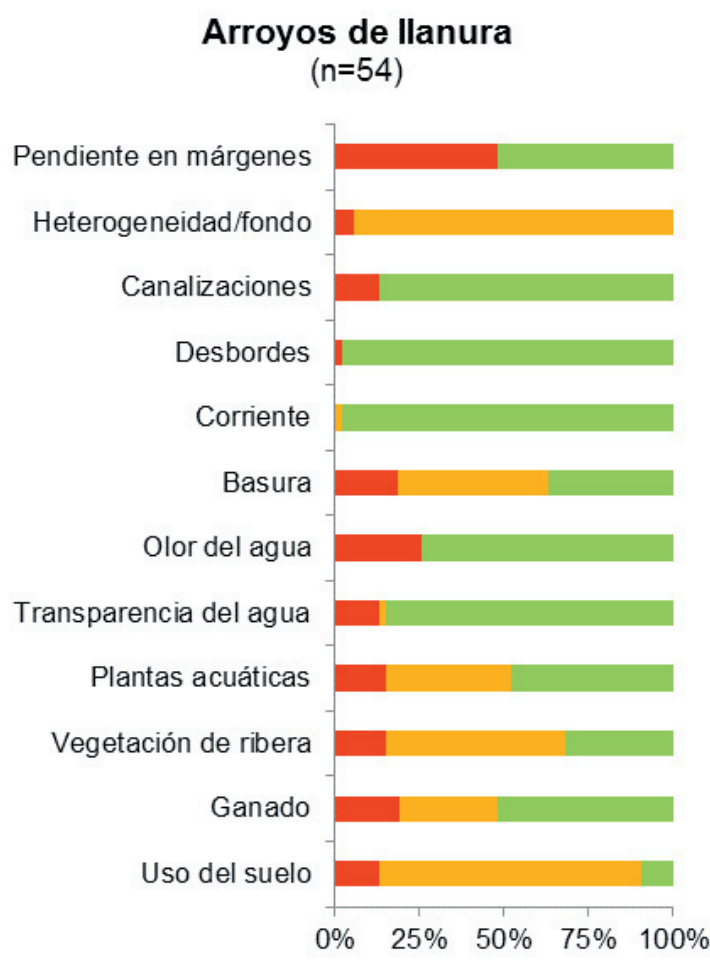

Estuarios

$(n=21)$

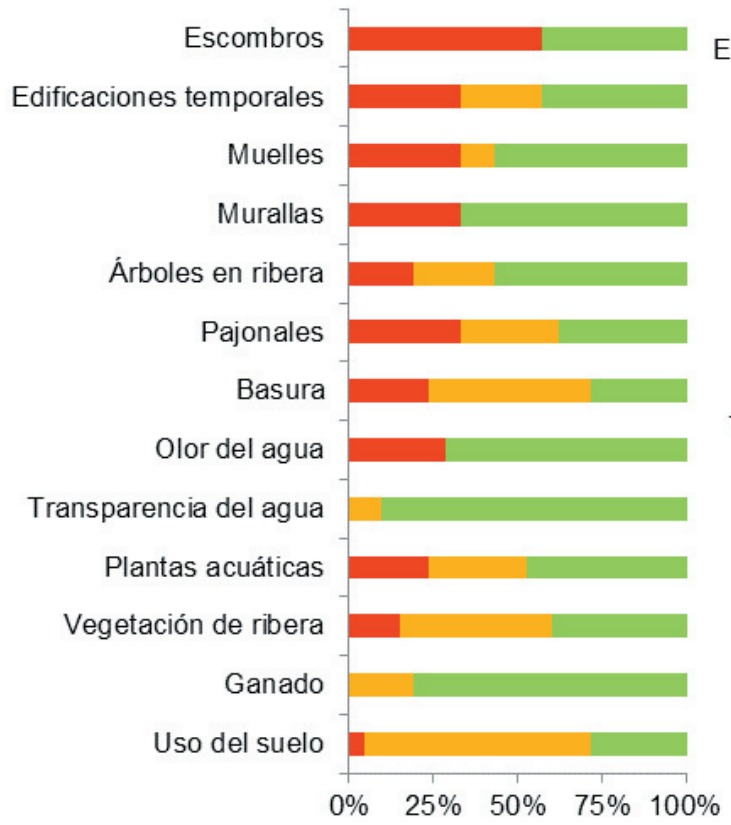

Arroyos de montaña

$(n=31)$

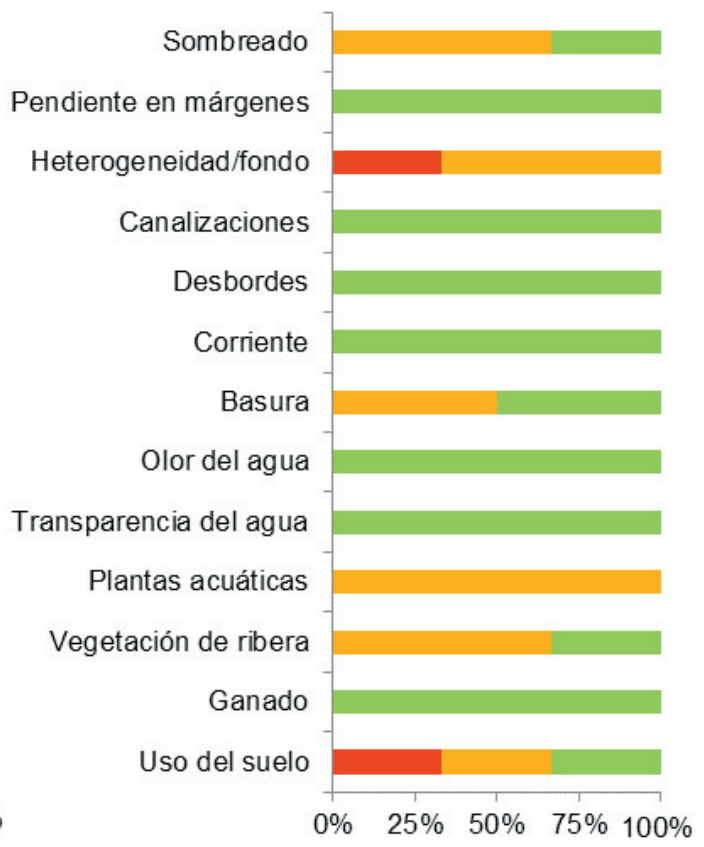

Lagunas

$(n=25)$

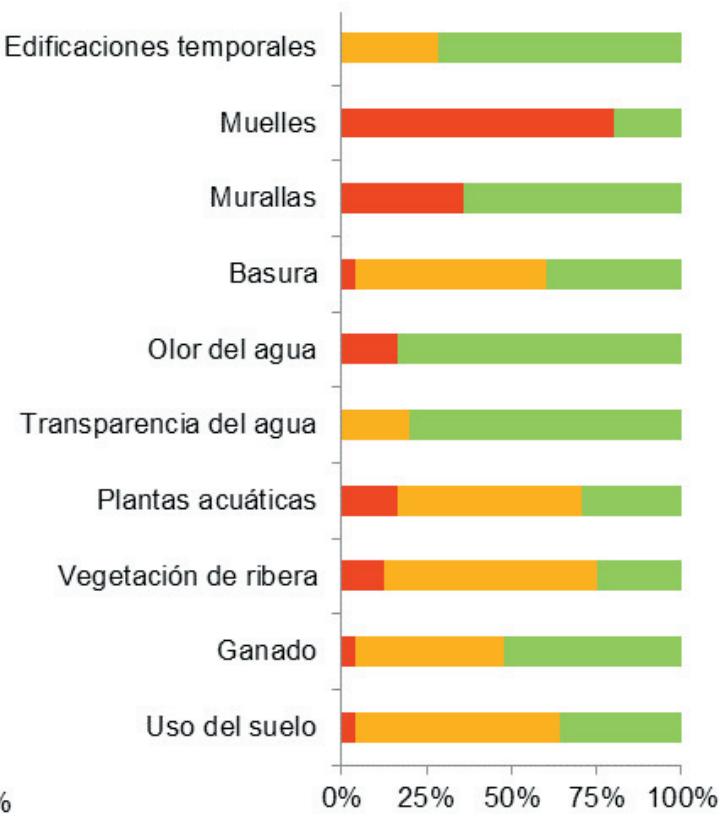

\section{- Malo $\quad$ Regular $\square$ Bueno}

Figura 4. Variables evaluadas en los cuatro tipos de ambientes, y su porcentaje de reportes malos, regulares y buenos según la apreciación de los ciudadanos científicos.

Figure 4. Evaluated variables for each of the four environments sampled by citizen scientists and their percentages of bad, regular and good reports. 
significativamente entre sí. La primera validación confirmó que las preguntas en las encuestas y la utilización de la aplicación en general son entendidas con la misma facilidad por aquellos usuarios con y sin formación científica. Este resultado es coherente si se considera que el desarrollo de la aplicación fue realizado aceptando las críticas y sugerencias de grupos de prueba con distinta formación académica y edad. Resultados similares comparando lo obtenido por profesionales del estudio del medioambiente y el público en general fueron reportados anteriormente en diversos tipos de estudios y representan una de los argumentos fundamentales a favor de la utilización de herramientas de ciencia ciudadana en estudios científicos (Irwin 1995; Cohn 2008; Theobald et al. 2015).

La validación de los resultados obtenidos por medio de la aplicación con datos ambientales tomados por métodos estandarizados y por personal técnico es una etapa fundamental para asegurar la calidad de los datos del proyecto. La segunda validación, realizada hasta el momento con los datos de ambientes de llanura y de estuario, muestra una correlación entre los valores de calidad del hábitat evaluados desde la aplicación por usuarios de distintos perfiles y los valores calculados para los mismos sitios a través de índices ecológicos de calidad del hábitat. Para ríos de llanura periurbanos, el índice del hábitat acuático USHI (Cochero et al. 2016) está correlacionado negativamente con las concentraciones de nitrógeno inorgánico disuelto y positivamente con las de oxígeno disuelto y con diversas variables biológicas (e.g., diversidad de diatomeas, concentración de clorofila-a, diversidad de macroinvertebrados, etc.). Los datos obtenidos a través de AppEAR indican una correlación con dicho índice, sugiriendo que las medidas obtenidas por los usuarios de la aplicación son consistentes en demostrar los deterioros en la calidad ecológica del hábitat. Una similar interpretación se puede realizar con los datos obtenidos para ambientes estuariales, que se correlacionaron de manera positiva con el índice IHRPlata (Gómez and Cochero 2013), un índice especialmente diseñado para indicar posibles deterioros de la costa del Río de la Plata.

El panorama de la ciencia ciudadana en Argentina es optimista. Proyectos de desarrollo nacional, como Flood Chasers (Le Coz et al. 2016), Caza Mosquitos (www.ilpla.edu.ar/ cazamosquitos) y los llevados a cabo por el grupo Cientópolis (www.cientopolis.org) demuestran un creciente interés por parte de científicos en el desarrollo y utilización de aplicaciones digitales de ciencia ciudadana para complementar sus proyectos. A nivel mundial, el éxito de proyectos tales como Ebird (con $>300.000$ usuarios y $>370$ millones de datos) o iNaturalist (con $>780.000$ usuarios y $>9$ millones de datos) lideran la vanguardia de las iniciativas de biomonitoreo gracias a la ciencia ciudadana; sus datos son cada vez más utilizados en la toma de decisiones y planes de manejo (Sullivan et al. 2009, 2017; NABCI Canada 2016; Ceccaroni and Piera 2017), y ayudan a alimentar bases de datos centralizadas de biodiversidad, como GBif (www.gbif.org). Todos estos proyectos plantearon problemáticas similares en su ejecución, inherentes a los proyectos de ciencia ciudadana (e.g., la verificación de la calidad de los datos, mantener el interés de los participantes, vincular los resultados a organismos oficiales, etc.), aunque se han incorporado cada vez más mecanismos para subsanarlos. Por ejemplo, se incorporaron sistemas de validación de dos etapas: en una primera instancia la validación de las fotografías por otros usuarios y luego, en una segunda instancia, la validación final la realiza un profesional (o curador, como se lo llama en iNaturalist). Esto permite que los ciudadanos científicos se involucren en la etapa de validación de datos del proyecto, y no sólo en la etapa de recolección de datos; esto le brinda más continuidad al proyecto y más calidad a sus datos.

En el caso de AppEAR, el principal enfoque del desarrollo actual del proyecto incluye comprobar y validar los datos obtenidos por los ciudadanos científicos con herramientas estandarizadas de monitoreo de la calidad del agua, en particular en lagunas y arroyos de montaña, donde no se realizaron dichas validaciones hasta el momento. También se planea adicionar la función de doble validación (por usuarios y por expertos). Esto ayudará a considerar a los datos obtenidos por ciudadanos científicos con rigurosidad para su utilización en estudios científicos a gran escala y por organismos de gestión. Futuras versiones del proyecto planean la incorporación de bioindicadores de fácil reconocimiento a las evaluaciones (e.g., macroinvertebrados, aves, peces), la ampliación de los ambientes a evaluar (e.g., lagos, costas marinas, etc.) y 


\section{AppEAR, CIENCIA CIUDADANA EN ECOSISTEMAS ACUÁTICOS}

la adecuación de los datos recolectados con bases de datos abiertas internacionales de biodiversidad (e.g., GBIF).

La aplicación AppEAR se puede descargar de manera libre y gratuita desde la plataforma de distribución digital de aplicaciones móviles Google Play. La cantidad de ciudadanos científicos que contribuyen con el proyecto y la cantidad de reportes que se envían sigue aumentando. Aunque es importante conocer y reconocer las limitaciones de la utilización de herramientas digitales masivas para la recolección de datos con fines científicos, se las debe entender como un complemento de las técnicas que se usan en los monitoreos tradicionales, que pueden enriquecer las distintas etapas de los estudios de investigación.

Agradecimientos. A cada ciudadano científico que ha participado de este proyecto, que con su entusiasmo y con sus aportes han hecho que este proyecto prolifere, colocando las palabras "ciencia ciudadana" en escuelas, laboratorios y medios de comunicación. Particularmente a Belén Sathicq por su incondicional ayuda y apoyo siete días a la semana; a Mercedes Nicolosi Gelis, Rocío Pazos, Santiago Tarda, Javier García de Souza y a todos los que acompañaron la realización de este proyecto desde el comienzo.

\section{REFERENCIAS}

Anbumozhi, V., J. Radhakrishnan, and E. Yamaji. 2005. Impact of riparian buffer zones on water quality and associated management considerations. Ecological Engineering 24:517-523.

Belmar, O., D. Bruno, F. Martínez-Capel, J. Barquín, and J. Velasco. 2013. Effects of flow regime alteration on fluvial habitats and riparian quality in a semiarid Mediterranean basin. Ecological Indicators 30:52-64.

Bowser, A., D. Hansen, J. Preece, Y. He, C. Boston, and J. Hammock. 2014. Gamifying citizen science. Proceedings of the companion publication of the 17th ACM conference on Computer supported cooperative work and social computing - CSCW Companion 14:137-140.

Burke, J., D. Estrin, M. Hansen, A. Parker, N. Ramanathan, S. Reddy, and M. B. Srivastava. 2006. Participatory Sensing.

Cardinale, B. J., M. A. Palmer, C. M. Swan, S. Brooks, and L. Poff. 2010. The Influence of Substrate Heterogeneity on Biofilm Metabolism in a Stream. America 83:412-422.

Carpenter, S. R., V. H. S. N. F. Caraco, D. L. Correll, R. W. Howarth, A. N. Sharpley, N. F. Caracco, D. L. Correll, R. W. Howarth, A. N. Sharpley, and V. H. Smith. 1998. Nonpoint pollution of surface waters with phosphorus and nitrogen. Ecological Applications 8:559-568.

Ceccaroni, L., and J. Piera. 2017. Analyzing the Role of Citizen Science in Modern Research.

Cochero, J., A. Cortelezzi, A. S. Tarda, and N. Gómez. 2016. An index to evaluate the fluvial habitat degradation in lowland urban streams. Ecological Indicators 71.

Cohn, J. P. 2008. Citizen Science: Can Volunteers Do Real Research? BioScience 58:192-197.

Conrad, C. C., and K. G. Hilchey. 2011. A review of citizen science and community-based environmental monitoring: Issues and opportunities. Environmental Monitoring and Assessment 176:273-291.

Conrad, C. T., and T. Daoust. 2008. Community-Based Monitoring Frameworks: Increasing the Effectiveness of Environmental Stewardship. Environmental Management 41:358-366.

Cooper, C. B., J. Dickinson, T. Phillips, and R. Bonney. 2007. Citizen science as a tool for conservation in residential ecosystems. Ecology and Society $\mathbf{1 2}$.

Le Coz, J., A. Patalano, D. Collins, N. F. Guillén, C. M. García, G. M. Smart, J. Bind, A. Chiaverini, R. Le Boursicaud, G. Dramais, and I. Braud. 2016. Crowdsourced data for flood hydrology: Feedback from recent citizen science projects in Argentina, France and New Zealand. Journal of Hydrology 541:766-777.

Daniels, R. B., and J. W. Gilliam. 1996. Sediment and Chemical Load Reduction by Grass and Riparian Filters. Soil Science Society of America Journal 60:246.

Danielsen, F., N. D. Burgess, P. M. Jensen, and K. Pirhofer-Walzl. 2010. Environmental monitoring: The scale and speed of implementation varies according to the degree of peoples involvement. Journal of Applied Ecology 47:1166-1168.

Dawson, F. H., and U. Kern-Hansen. 1979. The Effect of Natural and Artificial Shade on the Macrophytes of Lowland Streams and the Use of Shade as a Management Technique. Internationale Revue der gesamten Hydrobiologie und Hydrographie 64:437-455.

Dickinson, J. L., B. Zuckerberg, and D. N. Bonter. 2010. Citizen Science as an Ecological Research Tool: Challenges and Benefits. Annual Review of Ecology, Evolution, and Systematics 41:149-172.

Dris, R., H. Imhof, W. Sánchez, J. Gasperi, F. Galgani, B. Tassin, and C. Laforsch. 2015. Beyond the ocean: contamination of freshwater ecosystems with (micro-)plastic particles. Environmental Chemistry 12:539.

Fitzpatrick, M. C., E. L. Preisser, A. M. Ellison, and J. S. Elkinton. 2009. Observer bias and the detection of low-density populations. Ecological Applications 19:1673-1679.

Francis, R. A., and S. P. G. Hoggart. 2009. Urban river wall habitat and vegetation: observations from the River Thames through central London. Urban Ecosystems 12:465-485. 
Gómez, N., and J. Cochero. 2013. An index to assess the habitat quality in Southern Coastal Fringe of the Río de la Plata and its relations with other environmental indicators. Ecologia Austral 23.

González, M., and D. Tánago. 2011. Riparian Quality Index (RQI): A methodology for characterising and assessing the environmental conditions of riparian zones 29:235-254.

Haklay, M. 2015. Citizen Science and Policy: A European Perspective. Common Labs. Case Study Series 4:76.

Hupp, C. R. 1992. Riparian Vegetation Recovery Patterns Following Stream Channelization: A Geomorphic Perspective. Ecology 73:1209-1226.

Irwin, A. 1995. Citizen science: A study of people, expertise and sustainable development. Page Routledge.

Irwin, A. 2001. Constructing the scientific citizen: science and democracy in the biosciences. Public Understand Sci 10.

Kapp, K. M. 2012. The gamification of learning and instruction: game-based methods and strategies for training and education. Pfeiffer.

Klemann-Junior, L., M. A. Villegas Vallejos, P. Scherer-Neto, and J. R. S. Vitule. 2017. Traditional scientific data vs. uncoordinated citizen science effort: A review of the current status and comparison of data on avifauna in Southern Brazil. PloS one 12:e0188819.

Kruger, L. E., and M. A. Shannon. 2000. Getting to Know Ourselves and Our Places Through Participation in Civic Social Assessment. Society and Natural Resources 13:461-478.

Lake, P. S., M. A. Palmer, P. Biro, J. Cole, A. P. Covich, C. Dahm, J. Gibert, W. Goedkoop, K. Martens, and J. Verhoeven. 2000. Global change and the biodiversity of freshwater ecosystems: Impacts on linkages between above-sediment and sediment biota. BioScience 50:1099-1107.

Langhans, S. D., S. D. Tiegs, M. O. Gessner, and K. Tockner. 2008. Leaf-decomposition heterogeneity across a riverine floodplain mosaic. Aquatic Sciences 70:337-346.

Lavado Contador, J. F. 2005. Adaptive management, monitoring, and the ecological sustainability of a thermal-polluted water ecosystem: a case in SW Spain. Environmental monitoring and assessment 104:19-35.

Lepczyk, C. A., O. D. Boyle, T. L. Vargo, P. Gould, R. Jordan, L. Liebenberg, S. Masi, W. P. Mueller, M. D. Prysby, and H. Vaughan. 2009. Symposium 18: Citizen Science in Ecology: the Intersection of Research and Education. Bulletin of the Ecological Society of America 90:308-317.

Liddle, M. J., and H. R. A. Scorgie. 1980. The effects of recreation on freshwater plants and animals: A review. Biological Conservation 17:183-206.

Malmqvist, B., and S. Rundle. 2002. Threats to the running water ecosystems of the world. Environmental Conservation 29:134-153.

Milne, R., S. Rosolen, G. Whitelaw, and L. Bennett. 2006. Multi-party monitoring in Ontario: Challenges and emerging solutions. Environments 34:11-23

Miretzky, P., A. Saralegui, and A. F. Cirelli. 2004. Aquatic macrophytes potential for the simultaneous removal of heavy metals (Buenos Aires, Argentina). Chemosphere 57:997-1005.

Mishra, V. K., and B. D. Tripathi. 2008. Concurrent removal and accumulation of heavy metals by the three aquatic macrophytes. Bioresource Technology 99:7091-7097.

NABCI Canada. 2016. State of North America's birds.

Nürnberg, G. K. 1996. Trophic State of Clear and Colored, Soft- and Hardwater Lakes with Special Consideration of Nutrients, Anoxia, Phytoplankton and Fish. Lake and Reservoir Management 12:432-447.

Steelink, C. 1977. Humates and Other Natural Organic Substances in the Aquatic Environment. Journal of Chemical Education 54:599-603.

Steinberg, C. 2013. Ecology of humic substances in freshwaters: determinants from geochemistry to ecological niches. Springer Science and Business Media.

Strayer, D. L. 2006. Challenges for freshwater invertebrate conservation. Journal of the North American Benthological Society 25:271-287.

Sullivan, B. L., T. Phillips, A. A. Dayer, C. L. Wood, A. Farnsworth, M. J. Iliff, I. J. Davies, A. Wiggins, D. Fink, W. M. Hochachka, A. D. Rodewald, K. V. Rosenberg, R. Bonney, and S. Kelling. 2017. Using open access observational data for conservation action: A case study for birds. Biological Conservation 208:5-14.

Sullivan, B. L., C. L. Wood, M. J. Iliff, R. E. Bonney, D. Fink, and S. Kelling. 2009. eBird: A citizen-based bird observation network in the biological sciences. Biological Conservation 142:2282-2292.

Tabacchi, E., L. Lambs, H. Guilloy, A.-M. Planty-Tabacchi, E. Muller, and H. Décamps. 2000a. Impacts of riparian vegetation on hydrological processes. Hydrological Processes 14:2959-2976.

Tabacchi, E., L. Lambs, H. Guilloy, A.-M. Planty-Tabacchi, E. Muller, and H. Décamps. 2000b. Impacts of riparian vegetation on hydrological processes. Hydrological Processes 14:2959-2976.

Theobald, E. J., A. K. Ettinger, H. K. Burgess, L. B. DeBey, N. R. Schmidt, H. E. Froehlich, C. Wagner, J. HilleRisLambers, J. Tewksbury, M. A. Harsch, and J. K. Parrish. 2015. Global change and local solutions: Tapping the unrealized potential of citizen science for biodiversity research. Biological Conservation 181:236-244.

Tulloch, A. I. T., H. P. Possingham, L. N. Joseph, J. Szabo, and T. G. Martin. 2013. Realising the full potential of citizen science monitoring programs. Biological Conservation 165:128-138.

US EPA. 1997. Volunteer Stream Monitoring: A Methods Manual. Page (US EPA, ed.). URL: https:/ /www.epa.gov/ sites/production/files/2015-06/documents/stream.pdf. 


\section{AppEAR, CIENCIA CIUDADANA EN ECOSISTEMAS ACUÁTICOS}

Wagner, M., C. Scherer, D. Alvarez-Muñoz, N. Brennholt, X. Bourrain, S. Buchinger, E. Fries, C. Grosbois, J. Klasmeier, T. Marti, S. Rodriguez-Mozaz, R. Urbatzka, A. D. Vethaak, M. Winther-Nielsen, and G. Reifferscheid. 2014. Microplastics in freshwater ecosystems: what we know and what we need to know. Environmental Sciences Europe 26:12.

Whitelaw, G., H. Vaughan, B. Craig, and D. Atkinson. 2003. Establishing the Canadian Community Monitoring Network. Environmental Monitoring and Assessment 88:409-418. 